\title{
Ergänzungen zur Thüringer Käferfauna.
}

Von Pfarrer Wilhelm Hubenthal, Bufleben bei Gotha.

XIII.

Cicindela campestris L. v. connata Hr. 1 mit schwarzblauem violett schimmernden vergröfserten Augenpunkte der Flügeldecken. Neida, Bergholz (Sachsen-Altenburg). April 1918. 1 Stück. (K r m b olz, P o ls te r, Ent. Bl. 1918, 245.)

Miscodera arctica $\mathrm{Pk}$. Eisleben, Wiesengraben, 26. 6. 14 (K r a h n r t, Ent. Bl. 1918, 203).

Trechus subnotatus Dj. v. cardioderus Putz. Oberes Schleusetal, zwischen Allzunah und Gabel, Frauenwald gegenüber, am Bache anter einem Steine. 4. 8. 20. (R a p p.)

Acupalpus interstitialis Rttr. Fahner Höhe, 1921, 1 St., geschöpft. Bemerkenswertes Vorkommen dieser westdeutschen Art. (H e y $\mathrm{m}$ e s.)

Pterostichus lepidus Lske. a. ferreus Ltzn. Gotha, a. cyaneus Ltzn. Bufleben. a. niger Ltzn. Gotha.

Agonum atratum Dft. Klingenholz bei Tilleda. 25. 5. 77.

1 St. (P e tr y.)

Hydroporus 12-pustulatus F. Eisleben, Oberhüttenteich. 17. 5. 1909. (K rahnert.) (Ent. Bl. 1918, 203.) Nach R a p p auch von $\mathrm{E} g \mathrm{~g}$ e $\mathrm{r} \mathrm{s}$ bei Eisleben gefunden.

Colymbetes dolobratus Pk. wird von A p e tz, Beiträge zur Fauna des Osterlandes (Mitteilungen aus dem Osterlande. IV. 1840, p. 168-208) aus dem Osterlande angeführt. Diese Angabe ist nicht zu kontrollieren und jedenfalls irrtümlich. Auch aus Preufsen wurde er irrtümlich angeführt.

(B. E. Z. 1868 , p. 288.)

Micropeplus staphylinoides Mrsh. (von Ma a Is angeführt, D. E. Z. 1917, 121) ist in fulvus Er. zu ändern (vidi).

Anthobium torquatum Mrsh. Elgersburg, in Blüten von Spartium, s. h. 16. 5. 20. (L i e b m a n n.)

Phloeonomus lapponicus Zett. Sachsenburg. 19. 9. 18. Schmücke bei Heldrungen. 20.9.18 unter Kiefernrinde, mehrere St. (R a p p.)

Trogophloeus punctatellus Er. Nord-Thüringen, Stempeda, 13. 4. 17. 1 St. (P et r y.)

Bledius femoralis Gyll. Naumburg a. S., Schelbitzer Teiche. 23. 5. 19. 1 St. (M e r ten s.) - pygmaeus Er. Kyffhäuser, Kalkthal, 21. 10. 17. 1 St. (P etry.)

Oxyporus maxillosus F. Varietät: ganz gelb, nur Hinterecken der Flgd. geschwärzt: Friedrichroda, 1 St. (Grave, in coll. R a p p.) 
Stenus asphaltinus Er. (von $\mathrm{M}$ a a $\int \mathrm{s}$ angeführt, 1. c.) ist in canaliculatus Gyll. zu ändern (vidi). - cautus Er. Diese Art kommt in Thüringen vor, und nicht vafellus. Er. K e $11 \mathrm{ne} \mathrm{r} \mathrm{s} \mathrm{Verzeichnis}$ und Sammlung, sowie Sammlung Ma a fs, sind danach zu berichtigen. - brunnipes Stph. mit ganz schwarzen Beinen: Kyffhäuser, Kalkthal, 9.10. 16. 2 St. (P etry.)

Medon bicolor Olv. Eisleben, Seeburg. 22. 4. 16. 1 St. (Feige, vidi) (Ent. Bl. 1918, 204). Schon von Runde 1835 aus Thür. angeführt.

Lathrobium ripicola Czw. Naumburg a. S., Kr. Hufe, gesiebt, 7. 5. 19. 1 St. (M a e r ten s.)

Achenium depressum Grv. (M a $\int$ s l. c.) ist richtig (vidi).

Mycetoporus punctus Gyll. v. plagiellus Reitt. Fahner Höhe, geschöpft. V. 1921. 1 St. (H e y m es.)

Tachyporus atriceps Stph. v. signifer Pd. Rtt. Erfurt, Haarberg, 21. 3. 97. 1 St. Fahner Höhe, 13. 10. 1900. 1 St. (H b t h l.) corpulentus J. Shlb. Fahner Höhe. V. 1921. 2 St. unter Kiefern geschöpft. (H e y me s.) Das in der 9. Ergänzung (D. E. Z. 1914) erwähnte Stück wurde ebenfalls unter Kiefern geschöpft. Bei Dessau ebenso.

Tachinus fimetarius Grv. v. P e cirka e Reitt. Sättelstädt. 3. 6. 93.1 St. $\quad(\mathrm{Hb} \mathrm{th} \mathrm{l}$.)

Oligota parva Krtz. Eisleben, Mohrenapotheke, 28. 30. 8. 21 mehrfach, Komposthaufen, gesiebt. (K ühlhorn.)

Atheta nidicola Johansen (Danmarks rovbiller, 1914. J a n s s o n, Die Arthropodenfauna der Eichhörnchennester. Entom. Tidskr. 1919, 75-92), bei Kleinfahner in Elsternestern Mitte Mai 1921, häufig (He y mes). Diese in Schweden, Dänemark und

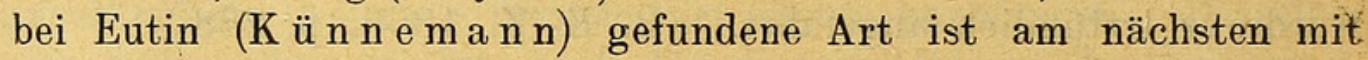
coriaria verwandt, hat aber stärkere Seitenborsten des nach vorn weniger verengten Halsschildes und stärkere Fühler. Das ठ̋ weicht durch den Mangel der grofsen Grube auf dem Halsschilde und durch andere Auszeichnung des letzten Tergites ab. Diese Art ist sicher überall in gröfseren Nestern zu finden. - ebenina Rey. Sachsenburg, gekötschert, IX. 1916. 1 St. (He y mes.)

Ityocara rubens Er. 24. 6. 03. 1 St. (J ä $\mathrm{nn}$ e r.)

Oxypoda Spaethi Bernh. Schwellenburg bei Erfurt, in der Nähe, von Kaninchenbauten geschöpft. 24. 8. 16. 1 St. (H e y mes, vidi, mit österreichischen Stücken übereinstimmend). - praecox Er. Kyffhäuser, Kattenburg, 4. 6. 16. 1 St. (Petry, vidi.) Brückners (1851) und K ellners (1867) Angaben gründen sich auf falsche Bestimmungen.

Euplectus Duponti Aub. Arnstadt, Altsiegelbacher Grund, aus 
Buchenlaub gesiebt. 4. 4. 20. 1 St. ( $\mathrm{Li} \mathrm{e} \mathrm{b} \mathrm{m} \mathrm{a} \mathrm{n} \mathrm{n.)} \mathrm{-} \mathrm{piceus}$ Mot. Altenburg. V. 20. 1 St. (H e y m e s.)

Neuraphes bescidicus Reitt. Walkenried. 1. 8. 20.1 St. (D orn.) - talparum Neresh. Arnstadt, Maulwurfsnester, mehrfach. (Li e b m a n n.)

Xylodrepa 4-punctata L. v. 6-punctata Gerh. Grimmenthal. 9. 5. 15. 1 St. (F i e d l e r.)

Liodes dubia Kug. v. longipes Schm. Stotternheim. VII. (F r ank.) 16. 8. 98.1 St. ( $1 \mathrm{bth}$ l.)

Anisotoma humeralis F. v. clavipes Hbst. Sachsenburg. VI. 1 St. (R a p p.)

Hister terricola Grm. v. amicorum Bickh. Ent. Bl. 1918, 228 (mit innerem Subhumeralstreifen). Thüringen. 2 St. (coll. B i ck h a r dt. "Ich nenne sie zu Ehren meiner Thüringer Freunde.") stigmosus Mars. Kyffhäuser. Kattenburg, in Pilz. 15. 7. 11. 1 St. (P e tr y.) (Schon 1. Ergänzung D. E. Z. 1902.)

Saprinus lautus Er. Naumburg a. S. Pöppern. 29. 5. 20. 1 St. (Ma e rten s.)

Gnathoncus punctulatus Thms. v. subsuturalis Reitt. Bufleben, im Hause. V. 21. 1 St. (Hbthl.)

Laccobius cinereus Mot. bei S chils k y (2. Aufl.) aus Thüringen usw. angeführt. Zu streichen. (cf. Ent. Bl. 1920, p. 245.) Nur aus dem Kaukasus und aus Sibirien bekannt.

Crenitis punctatostriata Letzn. Schmücke im Thür. Wald, Moortümpel. 20. 6. 20 in Mehrzahl. (L i e b m a n n.)

Cryptopleurum irenatum $\mathrm{Pz}$. Eisleben, Mohrenapotheke. 11.4. 14. 1 St. (F e ige, vidi.) (Ent. Bl. 1918, 205.)

Troglops cephalotes OIv., von $\mathrm{M}$ a a $\int \mathrm{s}$ angeführt (1. c.), ist in albicans L. zu ändern.

Haplocnemus nigricornis F. v. fuscitibia Muls. Friedrichroda, selten unter den häufigeren beiden anderen Färbungen. ( $\mathrm{G}$ r a v e.) 283.)

Trichodes apiarius L. v. apicida Kl. Thüringen. (D. E. Z. 1906 ,

Carpophilus decipiens Horn. Altenburg, im Hause, an Mehlkieister. 28.-29. 6. 20. 3 St. (H e y m es, vidi.)

Meligethes coeruleovirens Först. Stempeda 27. 6. 17. 2 St. (Petry, vidi.) - mbripes Mls. Thür. Wald. Gabel. VII. 20. 1 St. (H е у $\mathrm{m} \mathrm{es}$, vidi.) - bidens Bris. Sachsenburg, VIII. IX., einzeln, geschöpft. (H e y mes, vidi.) - sulcatus Bris. Fahner Höhe, Sommer 1921. 2 St., von Lamium geschöpft. (H e y m e s, vidi.)

Pocadius ferrugineus F. v. adustus Reitt. Arnstadt. (J u n g.)

Rhizophagus dispar v. punctulatus Gllb. Kickelhahn, 1. 9. 97. 1 St. $(\mathrm{Hb} \mathrm{h} \mathrm{l}$.) - bipustulatus F. v. Gyllenhali Thms. (nach M équignon zu dieser Art gehörend). Gotha. 1 St. (Hbthl.) 
Cybocephalus pulchellus Er. Kyffhäuser, Kosakenstein. 15. 4. 1914, 9. 10. 1916 je 1 St. (P etry.)

Cryptophagus domesticus Hbthl. (validus Ganglb. non Krtz.). Gotha, Haus, 1 St. Jena. 14. 5. 92.1 St. (H b th l.) (Ent. Bl. 1920, 241.) - punctipennis Bris. Reitt. non Ganglb. Nordhausen, Hamsterbau. 14. 9. 1908. 1 St. (Petry.) Bufleben, Maulwurfsgang, im Garten. ( $\mathrm{H} b \mathrm{thl}$.) Eisleben, See, Mäusenest. 30. 4. 21. (Kühlhorn.) Eisleben, Helfte, Maulwurfsnest. 21. 3. 21. (K ühlhorr.)

Enicmus brevicornis Mnnh. Sachsenburg. VI. 1 St. (R a p p.)

Hippodamia 7-maculata Deg. v. tarda Wse. Thür. Wald, Freibach. VIII. 1 St. (R a p p.)

Anisosticta 19-punctata L. v. conjuncta Wse. Wipfratal. 7. 9. 19.

2 St. (R a p p, L i e b m a n n.)

Coccinella 7-punctata L. v. turcica Wse. Naumburg, 1912. 1 St. (M a e r t e n s.) - lynacaea Olv. v. agnata Rosh. Rofsbach. 18. 4. 20. 1 St. (M a e r t e n s.)

Clitosthetus arcuatus Rssi. Eisleben, Alter Friedhof. 12.11. 13. 1 St. (Krahnert, vidi.) (Ent. Bl. 1918, 206.)

Helodes marginata F. v. nigricans Schls. 20.6. 20. Im oberen zahmen Geratal, nicht seIten unter Steinen, die fast vom Wasser bespült werden. (L i e b m a n n.)

Heterocerus sericans Ksw., von M a Is angegeben (1. c.), ist fusculus Ksw. (vidi).

Cytilus auricomus Dft. An feuchten Stellen, sicher weiter verbreitet; wird nicht erkannt, weil das Abdomen verklebt wird; 18. 5. 19. Im Geniste am Rande des Sumpfes zwischen Wachsenburg und Freudenthal. (R a p p, zuerst erkannt.) Auch von L i e b mann festgestellt. Willrode. 26. 4. 96. (M a a fs.)

Elater elongatulus F. v. balteatulus Reitt. Altenburg. V. 19. 3 St. VIII. 19. 1 St. (H e y m e s.)

Athous vittatus F. v. impallens Bss. Erfurt, Steiger. V. 1 St. (R a p p.)

Melanophila acuminata Deg. Eisleben, Krughütte. 10. 9. 19. 1 St. (K üh 1 h o r n.)

Anthaxia aurulenta F. Halle. (P anze r, F. Germ. V. 11.) (Buprestis aumulenta L. nicht in Thüringen!)

Agrilus pseudocyaneus Ksw. Kyffhäuser, Kattenburg, am Stamm von Populus tremula. 14. 7. 05. 1 St. (Petry, Reitte det., vidi.)

Trachys pygmaea F. Sachsenburg, auf Lavatera thuringiaca. (Schumacher, D. E. Z. 1919, 215.) Jedenfalls ist troglodytes Gyll. gemeint, die dort sicher vorkommt. Jenes Belegstück ist nicht erreichbar. Bei Brückner (1851) sicher auch falsch. 
Dryophilus anobioides Chevr. Suhl, von einer alten Laubholzhecke geklopft. 3. 5. 18 . 1 St. (F i e d le r.)

Ernobius densicornis Muls. Altenburg. VI. 19. 1 St. (H e y mes, vidi.) - Mulsanti Ksw. Fahner Höhe, geschöpft. 24. 6. 21. 1 St. (H e y $\mathrm{m} \mathrm{e} \mathrm{s,} \mathrm{vidi).}$

Anthicus minutus Laf. für Thüringen zu streichen (D. E. Z. 1908 , 43. 3. Ergänzung); das Stück ist humilis Grm. v. detritus Rey, welcher neu für Thür. ist. - hispidus Rssi. Eisleben, Wiesenpappel. 23. 10. 14. (F e ig e.) (Ent. Bl. 1918, 207.)

Tetratoma Desmaresti Latr. (schon in der zwölften Ergänzung angeführt) wurde am 22.9.16 in 1 St. am Kyffhäuser, Königsholz, gefunden. (P etry.)

Mycetoma suturalis Pz. (IV. 129). In der Originalbeschreibung: Göttingen. Ebenso bei $\mathrm{B}$ a $\mathrm{ch}$.

Gonocephalum pusillum F. Von $\mathrm{M}$ a a Is angeführt (1. c.), aber in seiner Sammlung keine Stücke aus Thür.

Oxymirus cursor L. v. nigricollis Ltzn. Friedrichroda, 2 St. (G r a ve.)

Evodinus clathratus v. signatus Pz. (V. 192): Göttingen, in Wäldern.

Gaurotes virginea L. v. violacea Deg. Thüringer Wald: Sieglitz VII. 1 St. (H bth 1.) Mordfleck. 23. 7. 19. 1 St. (M a a Is.) Leptura cerambyciformis Schrk. v. bisbistigma Pic. Blankenburg, Thür. Wald. v. 8-maculata Schall. Blankenburg, Freibach. v. Salbachi Reitt. Blankenburg (H b th 1.). - aethiops Pod. v. Letzneri Gabr. Thüringen, verbreitet. Fahner Höhe. (H e y mes, zuerst erkannt).

Leptidea brevipennis Mls. Von $\mathrm{M}$ a a $\int \mathrm{s}$ angeführt (l. c.); richtig. Aromia moschata L. v. cuprata Reitt. Gotha. (Rie m e r, 1 St. in meiner Sammlung.)

Criocephalus polonicus Mot. Eisleben, Landwehr 2, im Hause, 1. 7. 12. (Ehrhardt.) (Ent. Bl. 1918, 208.)

Exocentrus punctipennis Muls. im Parke von Weimar, fliegend. 1 St. 14. 8. 19. (F i e d l e r.)

Saperda 8-punctata Scop. Halle, auf Linden und Obstbäumen (P a n zer, V. 131). Auch bei Meiningen (nach B r ü ckner).

Clytra 4-punctata L. v. dissimilis Wse. Paulinzella. 21. 7. 19. 1 St. (J än n e r.) - appendicina Lac. Laucha a. Unstrut, nicht selten. (C. Schenkling.) Sachsenburg. V. VI. (Hbthl., Jänner.) Hörselberg. VI. (J änner.) Kyffhäuser. V. (Jänner.)

Cryptocephalus cristula Duf. Übergang zu v. violaceus Gerh. Erfurt, Henne. VIII. 1 St. (R a p p.) - ocellatus Drp. v. nigrifrons Bed. Stotternheim. VII. 1 St. (R a p p.) - bilineatus L. v. bisbilineatus Pic. Stotternheim. VII., gekötschert. (div. Samml.). 
Chrysomela carnifex F. v. coerulescens Suffr. Kyffhäuser. 7. 8. 09. 1 ㅇ. 15. 7. 11. 1 ऽ․ (P etry.) - geminata Pk. v. nigra Wse. Heuberg, Thür. Wald, V. 1 St. (schwarzblau). (R a p p.)

Phytodecta 5-punctatus F. v. aucupariae Jak. Friedrichroda (Grave.) - pallidus L. v. frontalis Olv. Friedrichroda. (G rave.)

Melasoma aeneum L. v. bicolor Schils. 1 St. aus Thüringen. (H b t h l.)

Chalcoides Plutus Latr. Eisleben, Wormsleben, 7.2.12 (Feige), 1 St. in meiner Sammlung.

Psylliodes aerea Fdr. (mit deutlicher flacher Schulterbeule). Alperstedt, zwei Stücke (M a $\int \mathrm{s}$ ) (als nov. sp. in meiner ersten Ergänzung, D. E. Z. 1902, 296). Alter Stollberg bei Nordhausen. 11. 7. 18, mehrfach (Petry); von Petry an Hey mes gegeben, der die Art erkannte. Nach $W$ e is es Tabelle kann man aerea wegen ihrer deutlichen Stirnlinien, welche denen der attenuata ähnlich sind, nicht bestimmen. Die von $\mathrm{Kell} \mathrm{n}$ e $\mathrm{r}$ angeführten aerea gehören zu picina. - napi F. v. Brisouti Bed. Kyffhäuser, Kalkthal, 9. 10. 16. 1 St. (P etry.)

Phyllotreta procera Rdtb. Bad Kösen, gestreift, wahrscheinlich von wilder Reseda. 25. 8. 19. (F i e d l e r.)

Longitarsus languidus Kutsch. Sachsenburg, sonnige Kalkhügel, geschöpft. IX. 1916. 1 St. (Heymes, vidi.) Auch die Exemplare $\mathrm{K}$ ell $\mathrm{ner}$ es sind doch diese Art (gegen meine Ansicht in D. E. Z. 1908, 270, dafs sie zu brunneus gehörten.)

Cassida splendidula Sffr. Kyffhäuser, Kalkthal. 21. 10. 17. 1 St. (Petry.) - vibex L. v. pannonica Sffr. Kyffhäuser, Kalkthal. 5. 6. 10. 1 St. (Petry.) Frankenhausen. 15. 8. 16. (F e i g e), 1 St. in coll. R a p p.

Acanthoscelides tessellatus Klug. (nomen in Mus. Berol.). Erfurt, in Benarys Gärtnerei, in Akaziensamen aus Ostindien. (Schröder.)

Laria ulicis Muls. Erfurt, in Benarys Gärtnerei, in Ervumsamen aus Südfrankreich. ( $\mathrm{S}$ ch r öd e r.)

Pachymerns chinensis L. von M a $\int \mathrm{s}$ angeführt (l. c.), ist richtig (vidi).

Otiorrhynchus laevigatus F. v. cornicinus St]. (unwesentliche Form). Meiningen, Dreifsigacker. (C. v. H e y de n.) Meiningen (S a a lmüller.) Die Typen mit dieser Bezeichnung in coll. v. H e y den. - raucus F. v. fulvus F. Sachsenburg. IV. 2 St. (Hbth 1.) - rugifrons Gyll. An den Gipsfelsen des Sachsensteins zwischen Walkenried und Sachsa. V. VIII., selten. (P etry, Ent. Bl. 1921, 92.)

Phyllobius calcaratus F. v. atrovirens Gyll. Bufleben, Pfarrgarten. V. 18.1 St. $(\mathrm{Hbth}$ 1.) 
Sitones intermedius Kürst. Kyffhäuser, Kalkthal. 9. 10. 16. 29. 4. 17. Je 1 S.t. (P et ry); hier nicht auf Coronilla, sondern auf Hippocrepis comosa, auf der an derselben Stelle auch Apion Curtisi und lanigerum vorkommen. (P e t r y.)

Trachyphloeus digitalis Gyll.! auf den Gipsbergen am südlichen Harzrande von Professor Petry in Nordhausen am Harz, der später genauere Angaben darüber veröffentlichen wird, in MehrzahI gefunden. Hey mes hat die Art zuerst erkannt; die Bestimmung ist jetzt sicher (vidi). - inermis Boh. Wormsleben bei Eisleben. 13. 4. 21. 1 St. (Kühlhorn, vidi.)

Coniocleonus cicatricosus Hppe. (callosus Bach). Auf diesen bezieht sich meine Angabe über crinipes Fahrs., der nur im Kaukasus und in Kleinasien vorkommt. Bach (Käferf. 2, 230) beschreibt nach seinem Unikum aus Boppard ganz deutlich dasselbe Tier, welches bei Wandersleben von Langenhan und später von mir bei der Sachsenburg gefangen wurde, erwähnt aber nichts von der auffallenden Behaarung der Beine. Bei crinipes Fahrs. ist diese Behaarung noch länger und kräftiger.

Lixus algirus L. Schon von $\mathrm{Z}$ e be und $\mathrm{M}$ ölle $\mathrm{r}$ für Thür. angegeben; später bezweifelt. Schmücke gegenüber Sachsenburg, gekötschert. 6. VII. 19. 1 St. (Rapp.) Dort von Ma a $\mathrm{s}$ öfters gefunden. Eisleben. ( $\mathrm{K} \ddot{\mathrm{u}} \mathrm{h} 1 \mathrm{~h}$ or $\mathrm{r}$.)

Tropiphorus ochraceosignatus Boh. Fahner. Höhe. V. 1921. 1 St. geschöpft. (H e y m e s.) Schon nach B a ch (vgl. Ergänzung 3 , p. 49) in Thüringen, aber bezweifelt, weil nicht mehr nachweisbar.

Liosoma cribrum Gyll. v. ruficolle Brnes. Gotha, Krahnberg. 31. 3. 88. 1 St. ( $\mathrm{Hbth}$ l.)

Hypera oxalidis Hbst. Suhl. VI. 1 St. (R a p p.)

Coeliodes dryados Gmel. v. infuscatus Neresh. Sachsenburg. 10. IX. 20. 1 St. (H e y m e s.)

Rhinoncus castor F. v. flavipes Stph. Suhl. 21. 6.12. 2 St. (F i e d l e r.)

Rhytidosoma fallax Otto. Suhl, gestreift. 20.5. 18. 1 St. (Fiedler.) Thür. Wald, Gabel, auf trockenen Feldrainen geschöpft. VII. 20. 2 St. (H e y m e s.)

Ceuthorrhynchus signatus Gyll. Kyffhäuser, Galgenberg. 18. 6. 16 . 1 St. (P e t r y.) Sachsenburg, Sommer 1920. (He y m e s, vidi.) Schon von $\mathrm{Bach}$ und $\mathrm{Z} \mathrm{e} \mathrm{be} \mathrm{angeführt} \mathrm{(1.} \mathrm{Ergänzung,} \mathrm{D.} \mathrm{E.} \mathrm{Z.}$ 1902.) - larvatus Schltze. Fahner Höhe, 6. 20, auf Anchusa officinalis und Pulmonaria officinalis mehrfach, auf Cynoglossum officinale ein Stück. (H e y m es, vidi.) Ornatus Gyll. in Sammlung $\mathrm{K}$ e $11 \mathrm{ner}$ und $\mathrm{M}$ a a $\mathrm{Ss}$ aus Thür. sind richtig! - K e 11 - 
ners rapae Gyll. besteht aus 1 rapae und 5 syrites. - griseus Bris. Meiningen. 28. 3. 09. 21. 6. 09. Je 1 St. (Fiedler.) - parvulus Bris. Suhl. 4. 6. 08. 1 St. (Fi edle r.) - turbatus Schltze. Arnstadt. VI. 20. (U r b a n.) Sachsenburg. 5. 6. 20. 1 St. (Heymes.) - suturellus Gyll. kommt nicht in Thüringen vor. Es handelt sich bei Kellners Angabe um suturellus Bach (Faun. Mitt. II, 350), welcher in scapularis Gyll. gehört (D. E. Z. 1883. 329). Das Thüringer Stück hat nach B a ch s Mitteilung (l. c.) B a $\mathrm{ch}$ von $\mathrm{K}$ e $1 \mathrm{ln}$ e r zur Bestimmung erhalten. Suturellus Gyll. überhaupt nicht in Dentschland, nur in Taurien. - Kellners ignitus Grm. ist nach seiner Sammlung carinatus Gyll. Ignitus nicht in Thüringen. - erysimi F. v. cyaneus Wse. Erfurt, Henne. VI. einzeln. (R a p p.) - Leprieuri Bris. v. Rübsaameni Kolbe. Weimar. (W e i s e.) Kleinfahner. (H e y m e s) (cf. Ent. Mitt. 1920, $77 \mathrm{ff}$.) - aeneicollis Grm. Nordhausen, Schinderrasen. 13. 6. 17. 2 St. (P e try.)

Anthonomus undulatus Gyll. In der Sammlung Kratz befindet sich 1 o aus Thüringen, von $\mathrm{K}$ e $l 1 \mathrm{ner}$ stammend, auf Glimmerplättchen aufgeklebt, welches $\mathrm{Kraatz}$ offenbar von $\mathrm{Ke} l \mathrm{ln}$ er zur Determination erhalten hatte. (Desbrochers det.) Das von mir früher zu dieser Art gezogene Stück aus Reinhardsbrunn (D. E. Z. 1908, 47) ist pedicularius L. v. Javeti Dbr.

Orchestes alni L. v. maculicollis Schils. Halle (Ha belmann, in meiner Sammlung). - foliorum Müll. Wandersleben, Gispersleben, n. s. - foliorum Müll. v. Sequensi Stl. Sättelstädt. 1 St. ( $\mathrm{Hbth} \mathrm{l.)} \mathrm{-} \mathrm{angustifrons} \mathrm{West.} \mathrm{(cf.} \mathrm{Hbthl}$. Int. Ent. Ztschr. Guben. XIII. 1920 , 205). In Kellners Sammlung unter foliorum. Finsterbergen. 7. 09, zahlreich. (R a p p.) Wandersleben. 8. 19. 2 St. Dietendorf (div. Samml.).

Rhamphus oxyacanthae Marsh. Hering D. E. Z. 1921, 126. In $\mathrm{K}$ e $11 \mathrm{n}$ e r s Sammlung sind 3 Stück gröfser, etwas glänzender, also offenbar pulicarius; 24 Stück sind klein, matter, also oxyacanthae. Bahndamm zwischen Gotha und Seebergen. 14. 6. 97, häufig; ich besitze davon nur noch 1 St. Näheres über die Futterpflanzen muls noch für hiesige Gegend festgestellt werden. (In meiner Sammlung ist oxyacanthae auch von Dessau, 30. 5. 97, 1 St., und von Hamburg 2 St.)

Cionus fraxini v. flavoguttatus Stl. Suhl. 27. 4. 09.2 St. (Fiedler.) Früher als v. rectangulus Hbst. von mir angeführt.

Magdalinus Weisei Schrein. Altenburg. VII. $19.1 \mathrm{St}$. (H e i m e s, vidi.)

Nanophyes globulus Germ. in $\mathrm{K}$ e $11 \mathrm{n}$ e r s Sammlung ist alles gracilis Rdtb. Globulus sehr selten, meist falsch bestimmt. - 
marmoratus Gze. v. muficollis Rey. Stotternheim. VIII., mehrfach. (R a p p.)

Apion decolor Desbr. v. austriacum Wagn. kommt nicht in Thür. vor. Die Angabe in den Supplementa Entomol. VII. 1918 p. 58 ist ein Schreibfehler; es soll Württemberg heifsen. hungaricum Dbr. Suhl. 26. 6. 12. 1 St. (F i ed l e r.) - flavimanum Gyll. Grimmenthal. 2. 7. 05. 1 St. (F i e d l e r.) lanigerum Grm. Schilskys Angabe (cf. 7. Ergänzung D. E. Z. 1912, 74) bezieht sich auf Exemplare, die $\mathrm{Ma}$ a $\mathrm{fs}$ und $\mathrm{Rapp}$ bei Erfurt am Steiger auf Hippocrepis fingen. (S chilsky det.) Kyffhäuser, Kalkthal. V. VIII. auf Hippocrepis comosa, selten. (P e tr y.)

Rhynchites aeneovirens Mrsh. v. virens Gabr. Erfurt, Steiger V. (R a p p.) Wandersleber Gleiche. V. 1 St. (L a $n g$ e $n$ han.) Myelophilus piniperda L. v. rubripennis Reitt. Gotha, Seeberg, Fahner Höhe. ( $\mathrm{Hb}$ b h l.)

Pityophthorus glabratus Eichh. Gotha, Seeberg, X. 18. 1 St. (H b th l.) Sachsenburg. 1. 8. 21. 1 St. (Kühlhorn.)

Aphodius granarius L. v. ragusamus Reitt. Gotha. 1 St. Siebleber Teich. 18. 4. 95. 1 St. ( $\mathrm{Hbthl}$.) - depressus Kug. v. nigripes Kriechb. Elgersburg. VI. 1 St. Freibach. VIII. 1 St. (R a p p, S ch midt det.)

Phyllopertha horticola L. v. ustulatipennis Villa. Elgersburg. VI. 1 St. (R a p p.)

Anisoplia subnuda Reitt. Halle a. S. Klickweg 5/6. 1920.

1 St. (M a ertens, vidi.)

Anomala aenea Deg. v. tricolor Schils. Arnstadt (coll. Ra p p). 


\section{$2 \mathrm{BHL}$ Biodiversity Heritage Library}

Hubenthal, Pfarrer Wilhelm. 1922. "Ergänzungen zur Thüringer Käferfauna." Deutsche entomologische Zeitschrift 1922(1), 67-75.

https://doi.org/10.1002/mmnd.48019220106.

View This Item Online: https://www.biodiversitylibrary.org/item/103380

DOI: https://doi.org/10.1002/mmnd.48019220106

Permalink: https://www.biodiversitylibrary.org/partpdf/236280

\section{Holding Institution}

Harvard University, Museum of Comparative Zoology, Ernst Mayr Library

\section{Sponsored by}

Biodiversity Heritage Library

\section{Copyright \& Reuse}

Copyright Status: Public domain. The BHL considers that this work is no longer under copyright protection.

This document was created from content at the Biodiversity Heritage Library, the world's largest open access digital library for biodiversity literature and archives. Visit BHL at https://www.biodiversitylibrary.org. 